\title{
Mean ergodicity for compact operators
}

\author{
by \\ Heydar Radjavi (Halifax), Ping-Kwan Tam (Hong Kong) \\ and KoK-KeOng TAN (Halifax)
}

\begin{abstract}
A mean ergodic theorem is proved for a compact operator on a Banach space without assuming mean-boundedness. Some related results are also presented.
\end{abstract}

1. Introduction. In the literature, mean ergodic theorems for linear operators usually deal with operators which are power bounded (see, e.g., $[\mathrm{YK}]$ and $[\mathrm{Z}])$. However, already in 1945, Hille $[\mathrm{H}]$ gave an example of an operator $T$ on $X=L_{1}[0,1]$ which is mean ergodic (i.e., the sequence of averages $\left(n^{-1} \sum_{j=1}^{n} T^{j} x\right)_{n=1}^{\infty}$ converges strongly for every $\left.x \in X\right)$ but not powerbounded. By the Banach-Steinhaus theorem, a necessary condition for mean ergodicity is mean-boundedness, i.e., $\sup _{n} n^{-1}\left\|\sum_{j=1}^{n} T^{j}\right\|<\infty$ (which is C-mean-boundedness in $[\mathrm{E}]$ ). Also the strong (resp. weak) convergence of $\left(n^{-1} \sum_{j=1}^{n} T^{j} x\right)_{n=1}^{\infty}$ clearly implies that $\left(n^{-1} T^{n} x\right)_{n=1}^{\infty} \rightarrow 0$ strongly (resp. weakly). In the treatment of mean ergodic theory in the book of Dunford and Schwartz [DS], the operator $T$ is assumed to be mean-bounded (Theorem VIII.5.1, p. 661), or the sequence $\left(n^{-1} T^{n}\right)_{n=1}^{\infty}$ is assumed to converge to zero weakly (Theorem VIII.8.3, p. 711). In 1985, Émilion [E] gave an example of a positive operator on $L_{p}(1<p<\infty)$ which is mean ergodic and not power-bounded; he also showed by an example (due to I. Assani) that mean-boundedness of a compact operator $T$ does not imply $\left(n^{-1}\left\|T^{n}\right\|\right)_{n=1}^{\infty}$ $\rightarrow 0$. More recently, Derriennic [D] constructed a mean ergodic operator $T$ on a Hilbert space such that $\left\|T^{n}\right\| \geq n$ for every positive integer $n$; moreover, $T^{*}$ is weakly mean ergodic (i.e., the averages converge weakly for every point of the Hilbert space) but not mean ergodic. Moreover, Yoshimoto $[\mathrm{Y} 1, \mathrm{Y} 2]$ obtained, under the assumption that $\left(n^{-w}\left\|T^{n}\right\|\right)_{n=1}^{\infty} \rightarrow 0$ (resp.

2000 Mathematics Subject Classification: Primary 47B07, 47A35.

Key words and phrases: compact operator, mean ergodic theorem, fixed point.

The work of P. K. Tam and K. K. Tan is partially supported by a research grant from The Chinese University of Hong Kong.

H. Radjavi and K. K. Tan are partially supported by research grants from NSERC of Canada. 
$\left(n^{-w} T^{n}\right)_{n=1}^{\infty} \rightarrow 0$ in the strong operator topology), the equivalence between the convergence of $C_{n}^{(\alpha)}[T]$ in the uniform operator norm (resp., in the strong operator topology) and that of the so-called Dirichlet methods (which generalize the Abel method), where $w=\min (1, \alpha)$, and $C_{n}^{(1)}[T]=n^{-1} \sum_{j=0}^{n-1} T^{j}$.

In this paper, under a fairly weak condition (cf. Proposition 2.1(4) below), we shall first obtain a general mean ergodic theorem for operators $T$ which are not necessarily mean-bounded nor satisfy $\left(n^{-1} T^{n}\right)_{n=1}^{\infty} \rightarrow 0$ on $X$ uniformly, operator strongly, or operator weakly (cf. also Proposition 2.2). We next obtain a mean ergodic theorem for compact operators on a Banach space, which need not be mean-bounded nor satisfy $\left(n^{-1} T^{n}\right)_{n=1}^{\infty} \rightarrow 0$ on $X$ uniformly, operator strongly or operator weakly (cf. Theorem 2.3 and its corollaries below). Finally, in Theorem 2.10, we present a relation between our condition and power-boundedness.

2. Ergodic theorems. If $(X,\|\cdot\|)$ is a normed space, we denote by $\mathcal{B}(X)$ the space of all bounded linear operators on $X$. If $A \in \mathcal{B}(X)$, then $x \in X$ is called a fixed point of $A$ if $A x=x$, and $\sigma(A)$ denotes the spectrum of $A$.

We begin with the following result:

Proposition 2.1. Let $(X,\|\cdot\|)$ be a (real or complex) normed space, $A \in \mathcal{B}(X)$ and $x \in X$. Denote by $I$ the identity operator on $X$, and $\overline{(I-A) X}$ the norm closure of $(I-A) X$ in $X$.

(1) If for some subsequence $\left(n_{k}\right)_{k=1}^{\infty}$ of $(n)_{n=1}^{\infty}, n_{k}^{-1} \sum_{j=1}^{n_{k}} A^{j} x \rightarrow 0$ weakly as $k \rightarrow \infty$, then $x \in \overline{(I-A) X}$.

(2) If $n^{-1} A^{n} x \rightarrow 0$ weakly as $n \rightarrow \infty$ and $n_{k}^{-1} \sum_{j=1}^{n_{k}} A^{j} x \rightarrow \bar{x}$ weakly as $k \rightarrow \infty$ for some subsequence $\left(n_{k}\right)_{k=1}^{\infty}$ of $(n)_{n=1}^{\infty}$, then $A \bar{x}=\bar{x}$, and $x-\bar{x} \in \overline{(I-A) X}$.

(3) If $x=(I-A) y$ with $n^{-1} A^{n} y \rightarrow 0$ weakly (resp. strongly) as $n \rightarrow \infty$, then $n^{-1} \sum_{j=1}^{n} A^{j} x \rightarrow 0$ weakly (resp. strongly) as $n \rightarrow \infty$.

(4) Suppose $n^{-1} A^{n} x \rightarrow 0$ weakly as $n \rightarrow \infty$ and for some subsequence $\left(n_{k}\right)_{k=1}^{\infty}$ of $(n)_{n=1}^{\infty}, n_{k}^{-1} \sum_{j=1}^{n_{k}} A^{j} x \rightarrow \bar{x}$ weakly as $k \rightarrow \infty$. If

$$
x-\bar{x}=(I-A) y \quad \text { with } \quad n^{-1}\left\|A^{n} y\right\| \rightarrow 0 \text { as } n \rightarrow \infty,
$$
then $\left\|n^{-1} \sum_{j=1}^{n} A^{j} x-\bar{x}\right\| \rightarrow 0$ as $n \rightarrow \infty$.

Proof. (1) Note that

$$
\begin{aligned}
(I-A) & \left(I+\frac{n_{k}-1}{n_{k}} A+\frac{n_{k}-2}{n_{k}} A^{2}+\ldots+\frac{1}{n_{k}} A^{n_{k}-1}\right) x \\
& =\left[I-\frac{1}{n_{k}}\left(A+A^{2}+\ldots+A^{n_{k}}\right)\right] x \rightarrow x \quad \text { weakly as } k \rightarrow \infty .
\end{aligned}
$$

The desired conclusion follows. 
(2) By our assumption, setting $x_{n}=n^{-1} \sum_{j=1}^{n} A^{j} x$, we have

$$
\begin{aligned}
A x_{n_{k}} & =\frac{1}{n_{k}} \sum_{j=2}^{n_{k}+1} A^{k} x=\frac{1}{n_{k}}\left[\left(\sum_{j=1}^{n_{k}} A^{k} x\right)+A^{n_{k}+1} x-A x\right] \\
& =x_{n_{k}}+\frac{1}{n_{k}}\left(A^{n_{k}+1} x\right)-\frac{1}{n_{k}} A x \rightarrow \bar{x} \quad \text { weakly as } k \rightarrow \infty .
\end{aligned}
$$

Since $A$ is also weakly continuous, $A x_{n_{k}} \rightarrow A \bar{x}$ weakly as $k \rightarrow \infty$; thus we conclude that $A \bar{x}=\bar{x}$. Therefore

$$
\frac{1}{n_{k}} \sum_{j=1}^{n_{k}} A^{j}(x-\bar{x})=\frac{1}{n_{k}} \sum_{j=1}^{n_{k}} A^{j} x-\bar{x} \rightarrow 0 \quad \text { weakly as } k \rightarrow \infty .
$$

By (1), $x-\bar{x} \in \overline{(I-A) X}$.

(3) Since

$$
\begin{aligned}
\frac{1}{n} \sum_{j=1}^{n} A^{j} x & =\frac{1}{n} \sum_{j=1}^{n} A^{j}(I-A) y=\frac{1}{n} \sum_{j=1}^{n} A^{j} y-\frac{1}{n} \sum_{j=1}^{n} A^{j+1} y \\
& =\frac{1}{n} A y-\frac{1}{n} A^{n+1} y,
\end{aligned}
$$

the desired conclusions hold.

(4) By (2), $A \bar{x}=\bar{x}$. Thus by (3), we have

$$
\frac{1}{n} \sum_{j=1}^{n} A^{j} x-\bar{x}=\frac{1}{n} \sum_{j=1}^{n} A^{j}(x-\bar{x}) \rightarrow 0 \quad \text { strongly as } n \rightarrow \infty .
$$

Proposition 2.2. Let $(X,\|\cdot\|)$ be a (real or complex) Banach space, $A \in \mathcal{B}(X)$ and $x \in X$. Suppose $I-A$ is one-to-one and has closed range, $\left\|A^{n} x\right\| / n \rightarrow 0$ as $n \rightarrow \infty$ and $n_{k}^{-1} \sum_{j=1}^{n_{k}} A^{j} x \rightarrow \bar{x}$ weakly as $k \rightarrow \infty$ for a subsequence $\left(n_{k}\right)_{k=1}^{\infty}$ of $(n)_{n=1}^{\infty}$. Then there exists $y \in X$ satisfying the condition (*) of Proposition 2.1 above, and $\left\|n^{-1} \sum_{j=1}^{n} A^{j} x-\bar{x}\right\| \rightarrow 0$ as $n \rightarrow \infty$.

Proof. By Proposition 2.1(2), $A \bar{x}=\bar{x}$ and $x-\bar{x} \in \overline{(I-A) X}$. As $\left\|A^{n} x\right\| / n \rightarrow 0$ as $n \rightarrow \infty$, we have $\left\|A^{n}(x-\bar{x})\right\| / n \rightarrow 0$ as $n \rightarrow \infty$. Since $I-A$ has closed range and is one-to-one, there is a (unique) $y \in X$ such that $(I-A) y=x-\bar{x}$. Since $(I-A) X$ is (closed in $X$, hence) a Banach space, by the open mapping theorem $(I-A)^{-1}:(I-A) X \rightarrow X$ is bounded. Thus

$$
\begin{aligned}
\frac{\left\|A^{n} y\right\|}{n} & =\frac{\left\|(I-A)^{-1} A^{n}(x-\bar{x})\right\|}{n} \\
& \leq\left\|(I-A)^{-1}\right\| \frac{\left\|A^{n}(x-\bar{x})\right\|}{n} \rightarrow 0 \quad \text { as } n \rightarrow \infty .
\end{aligned}
$$

By Proposition 2.1(4), $\left\|n^{-1} \sum_{j=1}^{n} A^{j} x-\bar{x}\right\| \rightarrow 0$ as $n \rightarrow \infty$. 
We now present our main result:

Theorem 2.3. Let $(X,\|\cdot\|)$ be a (real or complex) Banach space, $A \in$ $\mathcal{B}(X)$ be a compact operator and $x \in X$ be such that $\left\|A^{n} x\right\| / n \rightarrow 0$ as $n \rightarrow \infty$. If the sequence $\left(n^{-1} \sum_{j=1}^{n} A^{j} x\right)_{n=1}^{\infty}$ is bounded, then it converges strongly to a fixed point of $A$.

Proof. As $A$ is compact and the sequence $\left(n^{-1} \sum_{j=1}^{n} A^{j} x\right)_{n=1}^{\infty}$ is bounded, every subsequence of the sequence $\left(n^{-1} \sum_{j=2}^{n+1} A^{j} x\right)_{n=1}^{\infty}$ has a convergent subsequence. Because $\left\|A^{n} x\right\| / n \rightarrow 0$ and

$$
\frac{1}{n} \sum_{j=1}^{n} A^{j} x=\frac{1}{n} \sum_{j=2}^{n+1} A^{j} x+\frac{1}{n}\left(A x-A^{n+1} x\right),
$$

every subsequence of the sequence $\left(n^{-1} \sum_{j=1}^{n} A^{j} x\right)_{n=1}^{\infty}$ also has a convergent subsequence.

CASE 1: A has no non-zero fixed point. Let $\left(n_{k}\right)_{k=1}^{\infty}$ be any subsequence of $(n)_{n=1}^{\infty}$ and $\bar{x} \in X$ such that $n_{k}^{-1} \sum_{j=1}^{n_{k}} A^{j} x \rightarrow \bar{x}$ as $k \rightarrow \infty$. By Proposition $2.1(2), A \bar{x}=\bar{x}$. Since $A$ has no non-zero fixed point, we must have $\bar{x}=0$. It follows that $n^{-1} \sum_{j=1}^{n} A^{j} x \rightarrow 0$ as $n \rightarrow \infty$.

CASE 2: A has non-zero fixed points.

SubCASE 1. Suppose $X$ is a complex Banach space. Let $\sigma_{2}=\sigma(A) \backslash\{1\}$. Then there is a Riesz decomposition of $X=X_{1} \oplus X_{2}$, where $X_{1}$ and $X_{2}$ are closed $A$-invariant subspaces of $X, X_{1}$ is finite-dimensional, $\sigma\left(A_{1}\right)=\{1\}$ and $\sigma\left(A_{2}\right)=\sigma_{2}$, where $A_{j}=\left.A\right|_{X_{j}}$ for $j=1,2$. Clearly each $A_{j}$ is compact on $X_{j}$, and the projection $E_{j}$ on $X_{j}$ corresponding to the decomposition satisfies $E_{j} A=A E_{j}=A_{j} E_{j}$. Let $x=x_{1}+x_{2}$, where $x_{j} \in X_{j}$ for $j=1,2$. Then

$$
\begin{aligned}
& \frac{\left\|A_{k}^{n} x_{k}\right\|}{n}=\frac{\left\|A_{k}^{n} E_{k} x\right\|}{n}=\frac{\left\|E_{k} A^{n} x\right\|}{n} \leq\left\|E_{k}\right\| \frac{\left\|A^{n} x\right\|}{n} \rightarrow 0, \\
& \left\|\frac{1}{n} \sum_{j=1}^{n} A_{k}^{j} x_{k}\right\|=\left\|\frac{1}{n} \sum_{j=1}^{n} E_{k} A^{j} x\right\| \leq\left\|E_{k}\right\| \cdot\left\|\frac{1}{n} \sum_{j=1}^{n} A^{j} x\right\| .
\end{aligned}
$$

By Case 1 , we have $n^{-1} \sum_{j=1}^{n} A_{2}^{j} x_{2} \rightarrow 0$ as $n \rightarrow \infty$.

We shall now show that $A_{1} x_{1}=x_{1}$, hence $n^{-1} \sum_{j=1}^{n} A_{1}^{k} x_{1}=x_{1}$ for all $n \geq 1$. This will show that $\left(n^{-1} \sum_{j=1}^{n} A^{j} x\right)_{n=1}^{\infty}$ converges to $x_{1}+0 \in X_{1}+X_{2}$, which is a fixed point of $A$, thus completing the proof.

Indeed, it suffices to show that for any $m \times m$ cell $K$ (where $m \geq 2$ ) in the Jordan form of $A_{1}$, 


$$
K=\left(\begin{array}{ccccc}
1 & 1 & & & \\
& 1 & 1 & & \\
& & \ddots & \ddots & \\
& & & 1 & 1 \\
& & & & 1
\end{array}\right)
$$

and any $y=\left[y_{1}, y_{2}, \ldots, y_{m}\right]^{t} \in \mathbb{C}^{m}$ with $\left\|K^{n} y\right\| / n \rightarrow 0$, we have $y_{2}=$ $y_{3}=\ldots=y_{m}=0$, hence $K y=y$. To this end, for each $p \geq m$, let $K^{p} y=\left[k_{1}^{(p)}, k_{2}^{(p)}, \ldots, k_{m}^{(p)}\right]^{t}$. Then for each $j=1, \ldots, m$,

$$
k_{j}^{(p)}=y_{j}+\left(\begin{array}{c}
p \\
1
\end{array}\right) y_{j+1}+\ldots+\left(\begin{array}{c}
p \\
m-j
\end{array}\right) y_{m} .
$$

Since

$$
\frac{1}{p} k_{m-1}^{(p)}=\frac{1}{p} y_{m-1}+\frac{1}{p}\left(\begin{array}{c}
p \\
1
\end{array}\right) y_{m} \rightarrow 0 \quad \text { as } p \rightarrow \infty,
$$

we must have $y_{m}=0$. If $y_{m}=\ldots=y_{j}=0$ for $j \geq 3$, then since

$$
\begin{aligned}
\frac{1}{p} k_{j-2}^{(p)} & =\frac{1}{p} y_{j-2}+\frac{1}{p}\left(\begin{array}{c}
p \\
1
\end{array}\right) y_{j-1}+\ldots+\frac{1}{p}\left(\begin{array}{c}
p \\
m-j+2
\end{array}\right) y_{m} \\
& =\frac{1}{p} y_{j-2}+\frac{1}{p}\left(\begin{array}{c}
p \\
1
\end{array}\right) y_{j-1} \rightarrow 0 \quad \text { as } p \rightarrow \infty,
\end{aligned}
$$

we must have $y_{j-1}=0$. Thus by induction, $y_{2}=y_{3}=\ldots=y_{m}=0$, and we are done in Subcase 1.

Subcase 2. Suppose $(X,\|\cdot\|)$ is a real Banach space. Let $X_{\mathbb{C}}$ be the complexification of $X$ and let $A_{\mathbb{C}}$ be the complexification of $A$ (see e.g. [PS] or [ERT, pp. 118-119]). Then $n^{-1}\left\|A_{\mathbb{C}}^{n}(x, 0)\right\|=n^{-1}\left\|A^{n} x\right\| \rightarrow 0$ as $n \rightarrow \infty$ and the sequence $\left(n^{-1} \sum_{j=1}^{n} A_{\mathbb{C}}^{j}(x, 0)\right)_{n=1}^{\infty}=\left(n^{-1} \sum_{j=1}^{n} A^{j} x, 0\right)_{n=1}^{\infty}$ is bounded. By Subcase 1 , the sequence $\left(n^{-1} \sum_{j=1}^{n} A_{\mathbb{C}}^{j}(x, 0)\right)_{n=1}^{\infty}$ converges to a fixed point $(\bar{x}, 0)$ of $A_{\mathbb{C}}$. It follows that $\left(n^{-1} \sum_{j=1}^{n} A^{j} x\right)_{n=1}^{\infty}$ converges to $\bar{x}$ which is a fixed point of $A$.

It is clear that the conditions in Proposition 2.1(4) are satisfied if $x, X, A$ are as given in Theorem 2.3. We note also that as briefly mentioned previously, in [E] there is given an example of a real $2 \times 2$ matrix $A$ which, regarded as an operator on $X=\mathbb{R}^{2}$, satisfies $\sup _{n} n^{-1} \sum_{j=1}^{n}\left\|A^{j}\right\|<\infty$, but for some $x \in X$, the sequence $\left(n^{-1}\left\|A^{n} x\right\|\right)_{n=1}^{\infty}$ does not tend to 0 . The following theorem is an easy but interesting consequence of Theorem 2.3; for some related results, the reader is referred to $[\mathrm{BGM}]$.

Theorem 2.4. Let $(X,\|\cdot\|)$ be a (real or complex) Banach space and $A \in \mathcal{B}(X)$ be a compact operator. Let $x \in X$ be such that a subsequence of $\left(A^{n} x\right)_{n=1}^{\infty}$ is bounded. Then $\left(n^{-1} \sum_{j=1}^{n} A^{j} x\right)_{n=1}^{\infty}$ converges to a fixed point of $A$. 
Proof. By Theorem 4 in [ERT, pp. 117-118], the whole sequence $\left(A^{n} x\right)_{n=1}^{\infty}$ is bounded. The desired conclusion then follows readily from our Theorem 2.3.

In particular, we have the following result which is Theorem 2.1 of [TT]:

Corollary 2.5. Let $A$ be an $m \times m$ complex (respectively, real) matrix and $x$ be an $m \times 1$ complex (respectively, real) vector. If $\left(A^{n} x\right)_{n=1}^{\infty}$ has a bounded subsequence, then $\left(n^{-1} \sum_{j=1}^{n} A^{j} x\right)_{n=1}^{\infty}$ converges to a fixed vector of $A$.

We emphasize that the compact operator $A$ in Theorem 2.3 (respectively, in Theorem 2.4, and the $m \times m$ matrix $A$ in Corollary 2.5) is not assumed to be mean-bounded. Indeed, we shall provide in the following a simple example of a compact operator $A$ satisfying the conditions in Theorem 2.3 , Theorem 2.4 and Corollary 2.5 respectively, but which is not mean-bounded.

ExAmple 2.6. Let $X=\mathbb{R}^{3}$ or $\mathbb{C}^{3}$ and

$$
A=\left[\begin{array}{lll}
b & 0 & 0 \\
0 & c & 0 \\
0 & 0 & d
\end{array}\right]
$$

where $|b|<1,|c|=1,|d|>1$. Then $A$ is a compact operator on $X$ which is not power-bounded and not mean-bounded so that Theorem 1 in $[\mathrm{YK}]$ is not applicable. Let $x=[r, s, u]^{t}$. Then the sequence $\left\|A^{n} x\right\| / n \rightarrow 0$ as $n \rightarrow \infty$ if and only if $u=0$, if and only if $\left(A^{n} x\right)_{n=1}^{\infty}$ has a bounded subsequence; moreover, in that case, the sequence $\left(n^{-1} \sum_{j=1}^{n} A^{j} x\right)_{n=1}^{\infty}$ (is bounded and) converges to $\bar{x}$, where

$$
\bar{x}= \begin{cases}{[0, s, 0]^{t}} & \text { if } c=1 \\ 0 & \text { if } c \neq 1\end{cases}
$$

and $\bar{x}$ is a fixed point of $A$. Note that in the present example, the condition $u=0$ is even necessary for the boundedness of the sequence $\left(n^{-1} \sum_{j=1}^{n} A^{j} x\right)_{n=1}^{\infty}$.

We now consider the conditions (a) $A$ is power-bounded (i.e., $\sup _{n \geq 1}\left\|A^{n}\right\|$ $<\infty$ ), and (b) $\left\|A^{n}\right\| / n \rightarrow 0$ as $n \rightarrow \infty$. In general, (b) is strictly weaker than (a) (see, e.g., [S]). However, in [MZ, Theorem 3], it is shown that for a Riesz operator $A$ on a complex Banach space, (a) and (b) are equivalent. In Theorem 2.10 below we present a slightly more general result for a not necessarily Riesz operator. It also generalizes the result of Sz.-Nagy [N] from a compact operator on a complex Hilbert space to an operator more general than a Riesz operator on a real or complex Hilbert space. For related results for more restrictive classes of operators, we refer the reader to [Ze].

We will need (parts of) three lemmas which are of some independent interest. In the first lemma, we consider a real Banach space $(X,\|\cdot\|)$, 
and $A \in \mathcal{B}(X)$. Let $\left(X_{\mathbb{C}},\|\cdot\|_{\mathbb{C}}\right)$, and $A_{\mathbb{C}}$ be its complexification. If $\|\cdot\|$ is induced by an inner product $\langle\cdot, \cdot\rangle$ i.e., if $X$ is a real Hilbert space, then we let $\left(X_{\mathbb{C}},\langle\cdot, \cdot\rangle_{\mathbb{C}}\right)$ be the (Hilbert space) complexification of $X$; and $\|\cdot\|_{\mathbb{C}}$ is induced by $\langle\cdot, \cdot\rangle_{\mathbb{C}}$ (see, e.g., [PS] or [ERT, pp. 118-119]).

LEMma 2.7. We use the above notations.

(1) Let $(X,\|\cdot\|)$ be a real Banach space, and let $A \in \mathcal{B}(X)$. Then $A$ is power-bounded if and only if its complexification $A_{\mathbb{C}}$ is power-bounded. Moreover, $\left\|A^{n}\right\| / n \rightarrow 0$ as $n \rightarrow \infty$ if and only if $\left\|A_{\mathbb{C}}^{n}\right\| / n \rightarrow 0$ as $n \rightarrow \infty$.

(2) Let $X$ be a real Hilbert space, and $A \in \mathcal{B}(X)$. Then $A$ is similar to a contraction on $X$ if and only if its complexification $A_{\mathbb{C}}$ is similar to a contraction on $X_{\mathbb{C}}$.

Proof. (1) Since there is a positive constant $d$ such that for every positive integer $n,\left\|A^{n}\right\| \leq\left\|A_{\mathbb{C}}^{n}\right\| \leq d\left\|A^{n}\right\|$, the assertions are obviously true.

(2) Suppose $A$ is similar to a contraction on the real Hilbert space $X$, and let $S$ be an invertible operator in $\mathcal{B}(X)$ such that $\left\|S A S^{-1}\right\| \leq 1$. Let $T=S \times S$. Then $T^{-1}=S^{-1} \times S^{-1}$ in $\mathcal{B}(Y)$, and $\left\|T A_{\mathbb{C}} T^{-1}\right\| \leq 1$, so $A_{\mathbb{C}}$ is similar to a contraction.

Conversely, suppose $A_{\mathbb{C}}$ is similar to a contraction on $X_{\mathbb{C}}$. We shall show that $A$ is similar to a contraction on $X$. Indeed, let $W$ be an invertible operator in $\mathcal{B}\left(X_{\mathbb{C}}\right)$ such that $\left\|W A_{\mathbb{C}} W^{-1}\right\| \leq 1$. By the Riesz representation theorem and spectral theorem, there exists a positive operator $P \in \mathcal{B}(X)$ such that $\langle P y, P x\rangle=\operatorname{Re}\langle W(y, 0), W(x, 0)\rangle_{\mathbb{C}}$; here, $\operatorname{Re} z$ denotes the real part of the complex number $z$. Then $P$ is bijective, hence invertible in $\mathcal{B}(X)$. Now for each $x \in X$,

$$
\begin{aligned}
\left\|P A P^{-1} x\right\| & =\left\|W\left(A P^{-1} x, 0\right)\right\|_{\mathbb{C}}=\left\|W A_{\mathbb{C}} W^{-1} W\left(P^{-1} x, 0\right)\right\|_{\mathbb{C}} \\
& \leq\left\|W\left(P^{-1} x, 0\right)\right\|_{\mathbb{C}}=\left\|P\left(P^{-1} x\right)\right\|=\|x\| ;
\end{aligned}
$$

thus $\left\|P A P^{-1}\right\| \leq 1$ and $A$ is similar to a contraction on $X$.

Lemma 2.8. Let $(X,\|\cdot\|)$ be a (real or complex) Banach space, let $A \in$ $\mathcal{B}(X)$, and let $X_{j}, j=1,2$, be $A$-invariant closed subspaces of $X$ such that $X=X_{1}+X_{2}$. Let $A_{j}$ denote the restriction of $A$ to $X_{j}, j=1,2$. Then $A$ is power-bounded if and only if $A_{j}, j=1,2$, are power-bounded. Moreover, $\lim _{n \rightarrow \infty}\left\|A^{n}\right\| / n=0$ if and only if $\lim _{n \rightarrow \infty}\left\|A_{j}^{n}\right\| / n=0$ for $j=1,2$.

Proof. Since $A_{j}^{n}$ is the restriction of $A^{n}$ to $X_{j},\left\|A_{j}^{n}\right\| \leq\left\|A^{n}\right\|$ and the necessity of both assertions are obviously true. For the sufficiency, suppose first $X$ is a complex Banach space. Note that by [R, Theorem 5.20, p. 130], there exists a positive constant $r$ such that for each $x \in X$, there are $x_{j} \in X_{j}$, $j=1,2$, satisfying $x=x_{1}+x_{2}$ and $\left\|x_{1}\right\|+\left\|x_{2}\right\| \leq r\|x\|$. Hence for each 
positive integer $n$,

$$
\begin{aligned}
\left\|A^{n} x\right\| & \leq\left\|A_{1}^{n} x_{1}\right\|+\left\|A_{2}^{n} x_{2}\right\| \leq\left(\left\|A_{1}^{n}\right\|+\left\|A_{2}^{n}\right\|\right)\left(\left\|x_{1}\right\|+\left\|x_{2}\right\|\right) \\
& \leq\left(\left\|A_{1}^{n}\right\|+\left\|A_{2}^{n}\right\|\right) r\|x\|
\end{aligned}
$$

and the sufficiency of both assertions in the complex Banach space case follows readily.

Suppose now that $X$ is a real Banach space and each $A_{j}$ is powerbounded. For notational simplicity, let $Y=X_{\mathbb{C}}$ and $B=A_{\mathbb{C}}$ be their complexifications. Define $Y_{j}=X_{j} \times X_{j}$ for $j=1,2$. Then each $Y_{j}$ is a closed $B$-invariant subspace of $Y$, and $Y=X_{\mathbb{C}}=Y_{1}+Y_{2}$. Let $B_{j}$ be the restriction of $B$ to $Y_{j}, j=1,2$. Then $B_{j}=A_{j} \times A_{j}=\left(A_{j}\right)_{\mathbb{C}}$. By Lemma 2.7, each $B_{j}$ is power-bounded. By the preceding paragraph, $B$ is power-bounded. By Lemma 2.7 again, $A$ is power-bounded. Similarly the sufficiency of the other assertion is proved.

Lemma 2.9. Let $(X,\|\cdot\|)$ be a (real or complex) Hilbert space, $A \in$ $\mathcal{B}(X)$, and $X_{j}, j=1,2$, be A-invariant closed subspaces of $X$ such that $X=X_{1}+X_{2}$. Let $A_{j}$ denote the restriction of $A$ to $X_{j}, j=1,2$. Then $A$ is similar to a contraction on $X$ if and only if each $A_{j}$ is similar to a contraction on $X_{j}, j=1,2$.

Proof. (1) Suppose $X$ is a complex Hilbert space. By Paulsen's result $[\mathrm{P}$, Corollary 3.5], the lemma is equivalent to the assertion that $A$ is completely polynomially bounded if and only if each $A_{j}, j=1,2$, is completely polynomially bounded. To show the latter assertion, note that for every square matrix $\left[p_{l k}\right]$ of (complex) polynomials (of one variable), $\left[p_{l k}\left(A_{j}\right)\right]$ is a restriction of $\left[p_{l k}(A)\right]$, so the necessity is clear. For the sufficiency, let $c_{j}$, $j=1,2$, be constants such that for every square matrix $\left[p_{l k}\right]$ of polynomials, $\left\|\left[p_{l k}\left(A_{j}\right)\right]\right\| \leq c_{j}\left\|\left[p_{l k}\right]\right\|_{\infty}, j=1,2$.

Consider $\left[p_{l k}(A)\right]_{1 \leq l, k \leq n}$ as an operator on the direct sum $\widetilde{X}=\sum_{k=1}^{n} \oplus X$ of $n$ copies of $X$, and let $\widetilde{x}=\left[x^{(k)}\right] \in \widetilde{X}$ be arbitrary. As in Lemma 2.8 above, there is a positive constant $r$ (independent of $n$ and $\widetilde{x}$ ) and for each $k=1, \ldots, n$, there are $x_{j}^{(k)} \in X_{j}, j=1,2$, satisfying $x^{(k)}=x_{1}^{(k)}+x_{2}^{(k)}$ and $\left\|x_{1}^{(k)}\right\|+\left\|x_{2}^{(k)}\right\| \leq r\left\|x^{(k)}\right\|$. Hence

$$
\begin{aligned}
\left\|\left[x_{1}^{(k)}\right]\right\|+\left\|\left[x_{2}^{(k)}\right]\right\| & \leq\left(\sum_{k=1}^{n}\left\|x_{1}^{(k)}\right\|^{2}\right)^{1 / 2}+\left(\sum_{k=1}^{n}\left\|x_{2}^{(k)}\right\|^{2}\right)^{1 / 2} \\
& \leq 2^{1 / 2}\left[\sum_{k=1}^{n}\left(\left\|x_{1}^{(k)}\right\|^{2}+\left\|x_{2}^{(k)}\right\|^{2}\right)\right]^{1 / 2} \\
& \leq 2^{1 / 2}\left[\sum_{k=1}^{n} r^{2}\left\|x^{(k)}\right\|^{2}\right]^{1 / 2} \leq c\|\widetilde{x}\|
\end{aligned}
$$


where $c=2^{1 / 2} r$. Now we have

$$
\begin{aligned}
\left\|\left[p_{l k}(A)\right] \widetilde{x}\right\| & =\left\|\left[\sum_{k=1}^{n} p_{l k}(A) x^{(k)}\right]\right\|=\left\|\left[\sum_{k=1}^{n} \sum_{j=1}^{2} p_{l k}\left(A_{j}\right) x_{j}^{(k)}\right]\right\| \\
& =\left\|\sum_{j=1}^{2}\left[\sum_{k=1}^{n} p_{l k}\left(A_{j}\right) x_{j}^{(k)}\right]\right\| \\
& \leq\left\|\left[\sum_{k=1}^{n} p_{l k}\left(A_{1}\right) x_{1}^{(k)}\right]\right\|+\left\|\left[\sum_{k=1}^{n} p_{l k}\left(A_{2}\right) x_{2}^{(k)}\right]\right\| \\
& =\left\|\left[p_{l k}\left(A_{1}\right)\right]\left[x_{1}^{(k)}\right]\right\|+\left\|\left[p_{l k}\left(A_{2}\right)\right]\left[x_{2}^{(k)}\right]\right\| \\
& \leq\left\|\left[p_{l k}\left(A_{1}\right)\right]\right\|\left\|\left[x_{1}^{(k)}\right]\right\|+\left\|\left[p_{l k}\left(A_{2}\right)\right]\right\|\left\|\left[x_{2}^{(k)}\right]\right\| \\
& \leq \max \left(c_{1}, c_{2}\right)\left\|\left[p_{l k}\right]\right\|_{\infty}\left(\left\|\left[x_{1}^{(k)}\right]\right\|+\left\|\left[x_{2}^{(k)}\right]\right\|\right) \\
& \leq c \max \left(c_{1}, c_{2}\right)\left\|\left[p_{l k}\right]\right\|_{\infty}\|\widetilde{x}\|
\end{aligned}
$$

so that $\left\|\left[p_{l k}(A)\right]\right\| \leq c \max \left(c_{1}, c_{2}\right)\left\|\left[p_{l k}\right]\right\|_{\infty}$. Thus $A$ is completely polynomially bounded. So the lemma is proved in the complex Hilbert space case.

(2) Suppose $X$ is a real Hilbert space. Then as in Lemma 2.8, we consider the complexifications. With the notation therein and by Lemma 2.7, $A$ (respectively $A_{j}$ ) is similar to a contraction if and only if so is $B$ (respectively $B_{j}$ ). By (1) above, the desired conclusion follows readily.

TheOREM 2.10. Let $(X,\|\cdot\|)$ be a (real or complex) Banach space and let $A \in \mathcal{B}(X)$. Let $X_{1}, X_{2}$ be closed $A$-invariant subspaces of $X$ such that $X_{1}$ is finite-dimensional, $X=X_{1}+X_{2}$, and the spectral radius $r_{\sigma}\left(A_{2}\right)=$ $\lim _{n \rightarrow \infty}\left\|A_{2}^{n}\right\|^{1 / n}$ is less than 1 , where $A_{j}$ denotes the restriction of $A$ to $X_{j}$. Suppose $\left\|A^{n}\right\| / n \rightarrow 0$ as $n \rightarrow \infty$. Then $A$ is power-bounded. If $X$ is a Hilbert space, then $A$ is similar to a contraction on $X$.

Proof. (1) Suppose $X$ is a complex Banach space. By Lemma 2.8, $\lim _{n \rightarrow \infty}\left\|A_{j}^{n}\right\| / n=0$ for $j=1,2$. Since $A_{1}$ is compact, $A_{1}$ is power-bounded by [MZ, Theorem 3]. Since $r_{\sigma}\left(A_{2}\right)<1$ and $\left\|A_{2}^{n}\right\| \rightarrow 0$ as $n \rightarrow \infty, A_{2}$ is power-bounded. By Lemma 2.8, $A$ is power-bounded.

(2) Let $X$ be a real Banach space. As in Lemma 2.8, we consider the complexifications. Using the notations therein and by Lemma 2.7, each $Y_{j}$ is a $B$-invariant closed subspace of $Y, Y_{1}$ is finite-dimensional, $Y=Y_{1}+Y_{2}$, $\lim _{n \rightarrow \infty}\left\|B^{n}\right\| / n=0$, and $r_{\sigma}\left(B_{2}\right)=\lim _{n \rightarrow \infty}\left\|B_{2}^{n}\right\|^{1 / n}=\lim _{n \rightarrow \infty}\left\|A_{2}^{n}\right\|^{1 / n}$ $<1$. By (1) above, $B$ is power-bounded. By Lemma 2.7, $A$ is power-bounded.

(3) Let $X$ be a complex Hilbert space. Since $r_{\sigma}\left(A_{2}\right)<1$, by Rota's result [RO], $A_{2}$ is similar to a (proper) contraction on $X_{2}$. On the other hand, since $\left\|A_{1}^{n}\right\| / n \rightarrow 0$ as $n \rightarrow \infty, r_{\sigma}\left(A_{1}\right) \leq 1$. If $r_{\sigma}\left(A_{1}\right)<1$, then again by Rota's result $[\mathrm{RO}], A_{1}$ is similar to a (proper) contraction on $X_{1}$. If $r_{\sigma}\left(A_{1}\right)=1$, 
then the condition $\lim _{n \rightarrow \infty}\left\|A_{1}^{n}\right\| / n=0$ together with Jordan canonical form (since $X_{1}$ is finite-dimensional) implies that $A_{1}$ is diagonalizable, and $A_{1}$ is similar to a contraction on $X_{1}$. Therefore each $A_{j}$ is similar to a contraction on $X_{j}$ for $j=1,2$. By Lemma 2.9, $A$ is similar to a contraction on $X$.

(4) Finally, let $X$ be a real Hilbert space. As in (2) above, we have $Y=X_{\mathbb{C}}=Y_{1}+Y_{2}$ (all complex Hilbert spaces) with $Y_{1}$ finite-dimensional, $B=A_{\mathbb{C}}=B_{1}+B_{2}, r_{\sigma}\left(B_{2}\right)<1$. Thus by (3) above, $A_{\mathbb{C}}$ is similar to a contraction on $X_{\mathbb{C}}$. By Lemma $2.9, A$ is similar to a contraction on $X$.

We note that in Lemma 2.8, Lemma 2.9, and Theorem 2.10, the sum $X=X_{1}+X_{2}$ need not be a direct sum; in particular, when $X$ is a Hilbert space, the sum $X=X_{1}+X_{2}$ need not be an orthogonal (or direct) sum.

Theorem 2.10 implies readily the following result in which the case of a compact operator on a complex Hilbert space was proved by Sz.-Nagy in $[\mathrm{N}]$ :

Corollary 2.11. Let $A$ be a power-bounded compact operator (respectively, a Riesz operator) on a real or complex (respectively, complex) Hilbert space $H$. Then $A$ is similar to a contraction on $H$.

Acknowledgements. The authors would like to thank the referee for his/her many insightful suggestions for improvement of the paper. They are also grateful to Professor Jaroslav Zemánek for giving valuable comments and for providing several references.

\section{References}

[BGM] T. Bermúdez, M. González and M. Mbekhta, Operators with an ergodic power, Studia Math. 141 (2000), 201-208.

[D] Y. Derriennic, On the mean ergodic theorem for Cesàro bounded operators, Colloq. Math. 84/85 (2000), 443-455.

[DS] N. Dunford and J. T. Schwartz, Linear Operators, Part I, Interscience, New York, 1967.

[ERT] M. Edelstein, H. Radjavi and K. K. Tan, Boundedness stability properties of linear and affine operators, Taiwanese J. Math. 2 (1998), 111-125.

[E] R. Émilion, Mean-bounded operators and mean ergodic theorems, J. Funct. Anal. 61 (1985), 1-14.

[H] E. Hille, Remarks on ergodic theorems, Trans. Amer. Math. Soc. 57 (1945), 246269.

[MZ] M. Mbekhta et J. Zemánek, Sur le théorème ergodique uniforme et le spectre, C. R. Acad. Sci. Paris Sér. I Math. 317 (1993), 1155-1158.

[N] B. Sz.-Nagy, Completely continuous operators with uniformly bounded iterates, Magyar Tud. Akad. Mat. Kutato Int. Közl. 4 (1959), 89-93.

[PS] D. Pascali and S. Sburlan, Nonlinear Mappings of Monotone Type, Editura Academiei, Bucureşti, 1978.

[P] V. I. Paulsen, Every completely polynomially bounded operator is similar to a contraction, J. Funct. Anal. 55 (1984), 1-17. 
[RO] G.-C. Rota, On models for linear operators, Comm. Pure Appl. Math. 13 (1960), 468-472.

[R] W. Rudin, Functional Analysis, McGraw-Hill, New York, 1973.

[S] A. Swięch, A note on the difference of the consecutive powers of operators, in: Linear Operators, Banach Center Publ. 38, Inst. Math., Polish Acad. Sci., 1997, 381-383.

[TT] P. K. Tam and K. K. Tan, A mean ergodic theorem on vector space, Appl. Math. Lett. 12 (1999), 61-64.

[Y1] T. Yoshimoto, Uniform and strong ergodic theorems in Banach spaces, Illinois J. Math. 42 (1998), 525-543; Correction, ibid. 43 (1999), 800-801.

[Y2] - Dirichlet series and uniform ergodic theorems for linear operators in Banach spaces, Studia Math. 141 (2000), 69-83.

[YK] K. Yosida and S. Kakutani, Operator theoretical treatment of Markov's process and mean ergodic theorem, Ann. of Math. 42 (1941), 188-228.

[Z] R. Zaharopol, Mean ergodicity of power-bounded operators in countably order complete Banach lattices, Math. Z. 192 (1986), 81-88.

[Ze] J. Zemánek, On the Gelfand-Hille theorem, in: Functional Analysis and Operator Theory, Banach Center Publ. 30, Inst. Math., Polish Acad. Sci., 1994, 369-385.

Department of Mathematics and Statistics

Dalhousie University

Halifax, Nova Scotia, Canada, B3H 3J5

E-mail: radjavi@mscs.dal.ca kktan@mscs.dal.ca
Department of Mathematics Chinese University of Hong Kong Shatin, New Territories, Hong Kong E-mail: pktam@math.cuhk.edu.hk

Received December 7, 1998

Revised version January 23, 2003 